Brain, Behavior and Evolution
Brain Behav Evol 2010;76:3-10

DOI: $\underline{10.1159 / 000320218}$
Received: July 15, 2010

Returned for revision: July 27, 2010 Accepted after revision: August 11, 2010

Published online: September 30, 2010

\title{
On the Origin and Evolution of the Tripartite Brain
}

\section{Frank Hirth}

King's College London, MRC Centre for Neurodegeneration Research, Department of Neuroscience, Institute of

Psychiatry, London, UK

\section{Key Words}

Brain - Nervous system $\cdot$ Homology $\cdot$ Phylogeny .

Evolution $\cdot$ Urbilateria $\cdot$ Otx $\cdot$ Emx $\cdot$ Engrailed $\cdot$

Functional equivalence

\begin{abstract}
The many different nervous systems found in bilaterally symmetric animals may indicate that the tripartite brain appeared several times during the course of bilaterian evolution. However, comparative developmental genetic evidence in arthropods, annelids, urochordates, and vertebrates suggests that the development of a tripartite brain is orchestrated by conserved molecular mechanisms. Similarities in the underlying genetic programs do not necessarily reflect a common origin of structures. Nevertheless, 3 lines of evidence support a monophyletic origin of the tripartite brain and possibly also an elongated central nervous system (CNS): structural homology, character identity networks, and the functional equivalence of character identity genes. Monophyly of the brain also implies that the brain was secondarily reduced and lost multiple times during the course of evolution, leading to extant brainless bilaterians. The likelihood of secondary loss can be estimated by metazoan divergence times and through reconstructed cases such as limb loss in tetrapods or eye loss in fish. When scaled to molecular clock dates, monophyly of the tripartite brain indi-
\end{abstract}

cates that existing brainless Bilateria had several hundred million years' time for the secondary modification and eventual loss of a primitive/ancestral brain and CNS. To corroborate this conjecture, ancestral character identity genes of living brainless Bilateria can be tested for their potential to substitute Drosophila or Mus homologs in tripartite brain development.

Copyright $\odot 2010$ S. Karger AG, Basel

Current cladistics is a mixture of Linnean taxonomy and the comparative analysis of morphological characters, developmental genetics, and phylogenomics. It supports a monophyletic origin of bilaterally symmetric animals and suggests a common ancestor called Urbilateria. The current view of evolutionary relationships in the animal kingdom, however, does not solve several longstanding questions and problems. One of these problems concerns the origin and evolution of the brain and central nervous system (CNS): how is it possible to reconcile monophyly with the many different shapes and types of nervous systems found in extant Bilateria? The problem is most obvious when the complex brains of insects, cephalopods, and mammals are compared with the nerve nets of echinoderms or brachiopods, or when the ventrally located nerve cord of arthropods is compared with the dorsally located nerve cord of vertebrates. Taken at face val-

\section{KARGER}

Fax +4161306 1234 E-Mail karger@karger.ch www.karger.com (c) 2010 S. Karger AG, Basel

0006-8977/10/0761-0003\$26.00/0

Accessible online at:

www.karger.com/bbe
Dr. Frank Hirth

King's College London, MRC Centre for Neurodegeneration Research

Department of Neuroscience, Institute of Psychiatry

PO Box 37, 16 De Crespigny Park, London SE5 8AF (UK)

Tel. +44 207848 0786, Fax +44 207708 0017, E-Mail Frank.Hirth@ kcl.ac.uk 
ue, these obvious differences can lead to the notion that complex, centralized nervous systems appeared independently several times during the course of bilaterian evolution and that the last common ancestor most likely possessed an uncentralized, possibly nerve net-like nervous system.

This is in essence the argument of Moroz [2009], which is also addressed by Northcutt [2010]. Its basic concept has been discussed previously [Meinhardt, 2002; Holland, 2003], but Moroz [2009] not only considers distinct morphological types of nervous organization, he also considers multiple, independent evolutionary origins of neurons. In support of the latter, Moroz [2009] argues that (i) no 'universal neuronal marker' has been identified; (ii) differences exist between neural specification in anthozoans and hydrozoans (cnidarians); (iii) protostomians, e.g. mollusc CNSs, can develop from different and unrelated body regions; (iv) nervous system-patterning genes are not exclusively expressed in neurons; (v) not all neurons originate in the epidermis, and (vi) multiple regulatory networks are independently recruited to establish complex sensory structures. Hence, Moroz [2009] proposes a polyphyletic origin of neurons, centralized nervous systems, and brains of bilaterally symmetric animals. He contrasts his hypothesis with the concept of a monophyletic origin of the brain and CNS which suggests that the last common bilaterian ancestor most likely possessed a complex, centralized nervous system with a tripartite brain [Hirth et al., 2003; Hirth and Reichert, 2007].

Moroz [2009] clearly emphasizes the need for more comparative developmental genetic and phylogenomic data, especially for taxa showing a radically different nervous organization as compared to that of standard laboratory animals such as flies and mice. These data are necessary in particular for taxa like onychophorans, as well as molluscs and brachiopods, as they represent crucial sister groups to arthropods and annelids, respectively. However, Moroz's [2009] argument neglects the fact that differences in the shape, size, and function of structures do not necessarily reflect differences in their genealogy, and that structural homologies do not need to correlate with similar genetic toolkits that underlie their development [Wagner et al., 2007; Carroll, 2008; Shubin et al., 2009]. The real problem with concepts about Urbilateria is that they are rather speculative and hinge on data feeding the cladogram where evidence refers to sister and out groups (see below).

The pros and cons and specific details of this debate have been recently reviewed [Hirth and Reichert, 2007;
Arendt et al., 2008; De Robertis, 2008; Hejnol and Martindale, 2008]. Therefore, I will only briefly summarize the major body of evidence supporting a monophyletic origin of the tripartite brain, and then discuss 2 major challenges to this concept: (i) the significance of sister and out groups used to establish monophyly of bilaterians and (ii) the secondary, independent loss of a tripartite brain and complex CNS multiple times during the course of evolution. I will not address another related conundrum, namely the seemingly inverted CNS of arthropods and chordates. New data suggest that the origin and evolution of the bilaterian mouth, anus, and through gut are less clear and definite than previously thought [Christiaen et al., 2007; Martindale and Hejnol, 2009]. These determinants are used to classify Protostomia and Deuterostomia $(\mathrm{P}+\mathrm{D})$, and to position a dorsoventral (DV) body axis which in turn is used to define the orientation of an elongated CNS as either dorsally or ventrally located [Lowe et al., 2006; Nomaksteinsky et al., 2009].

\section{Monophyly of the Tripartite Brain}

Comparative developmental genetic analyses in arthropods [Hirth et al., 2003], annelids [Denes et al., 2007], urochordates [Wada et al., 1998], and vertebrates [Boyl et al., 2001a] provide experimental evidence suggesting that brain and CNS development in these taxa is orchestrated by conserved molecular genetic mechanisms. As stated earlier, similarities in the underlying developmental genetic programs do not necessarily reflect a common origin of structures. However, monophyly of the tripartite brain is supported by 3 lines of evidence, namely (i) structural homology, (ii) character identity networks (ChINs), and (iii) functional equivalence of character identity genes.

\section{Structural Homology}

Homology signifies common descent and can be defined as a relationship between traits of organisms that are shared as a result of common ancestry [Mindell and Meyer, 2001]. Structural homology refers to a morphological character that is (a) derived from a common ancestor possessing this character, (b) built on the same basic plan, and (c) consists of comparable elements [Lorenz, 1974]. The latter was already exemplified by Darwin [1859], who referred to 'the relative position or connection in homologous parts; they may differ to almost any extent in form and size, and yet remain connected together in the same invariable order'. Textbook examples 
are the different forelimbs of tetrapods where similar bones are connected in the 'same invariable order', irrespective of the different functions they serve. The same principle of 'relative position or connection in homologous parts' applies to 'midbrain' structures in Drosophila and $M u s$, although function seems to be conserved as well; in both cases, GABAergic, serotonergic, and dopaminergic neural circuit elements involved in the control of locomotor behavior are located in the same relative position to other neural processing centers, namely posteriorly to light-sensing organs and anteriorly to gustatory and 'facial' innervation [Travers et al., 1987; Hanesch et al., 1989; Goridis and Rohrer, 2002; Prakash and Wurst, 2004; Kozmik, 2005; Melcher and Pankratz, 2005; Nichols, 2007; Neuser et al., 2008; Kong et al., 2010].

The principle 'connected together in the same invariable order' of comparable elements may also apply to ventral/spinal cord motor neurons in Drosophila, Platynereis (Annelida), and Mus; they are located ventrally to nonneural tissue and dorsally to the midline [Thor and Thomas, 2002; Arendt et al., 2008]. As a stand-alone criterion, however, 'relative position or connection in homologous parts' would be insufficient to signify homology because similar structures with similar positions can have multiple causes, including parallel or convergent evolution [Mindell and Meyer, 2001]. However, related to brain evolution, this criterion is supported by developmental genetic evidence suggesting that insect and mammalian brains are built on the same basic plan [Hirth and Reichert, 1999], which is executed by the spatiotemporal activities of pleiotropic genes that constitute character identity networks (ChINs).

\section{Character Identity Networks}

ChINs refer to genetic regulatory networks that control the developmental program and, hence, the specification of character identities, such as forelimbs [Wagner, 2007]. ChINs for brain and CNS development include Otx-Pax-Hox modules acting along the anterior-posterior axis, $B M P-M s x-N k x$ modules acting along a DV axis, and bHLH-Par-Numb modules acting along an apicobasal axis. Together, these modules are necessary for the correct formation of an orthogonal, species-specific CNS. Character identity genes are central to ChINs; their knockdown/mutational inactivation does obstruct the formation of a character identity. The majority of character identity genes are transcription factors, with textbook examples such as Pax6 genes, which are essential for the formation of light-sensing organs [Shubin et al., 2009], or otd/Otx genes, which are essential for anterior brain for- mation [Hirth and Reichert, 1999]. As stated earlier, similarities in developmental genetic programs do not necessarily reflect a common origin of character identities, which is exemplified, for example, by the role of distal-less $(D l l / D l x)$ genes in the formation of non-homologous appendages [Panganiban et al., 1997]. However, the situation is different if taxa-specific ChINs, which comprise homologous genes, specify taxa-specific character identities to which structural homology applies, namely their construction depends on a conserved genetic program (basic plan) and consists of comparable elements that are arranged 'in the same invariable order'. This principle applies to the above-mentioned midbrain-derived neural circuit elements that are required for the control of locomotor behavior: in both flies and mice, FGF8, Engrailed, and $\mathrm{Pax} 2 / 5 / 8$ genes are essential for the formation and specification of these structures [Wurst and Bally-Cuif, 2001; Ludlow, White and Hirth, submitted].

\section{Functional Equivalence of Character Identity Genes}

ChINs of different taxa can comprise homologous genes that control the developmental program and, hence, the specification of character identities sharing a common descent. It has therefore been predicted that the phenotype caused by knockdown of a character identity gene (i.e. transcription factor) 'can be reversed with a gene from the clade that shares this character, but not by genes from species that diverged before the origin of this character' [Wagner, 2007]. Experimental evidence conforming to this prediction is available. Human Otx2 can rescue defective 'forebrain' formation in Drosophila orthodenticle (otd) mutants [Leuzinger et al., 1998], and otd can replace mouse $0 t \times 2$ in fore- and midbrain formation, but only if otd is accompanied by the Ot $x 2$ regulatory sequences required for epiblast-specific translational control [Acampora et al., 2001; Boyl et al., 2001b]. Drosophila engrailed can substitute for mouse Engrailed 1 function in mid-hindbrain formation, but not for limb development [Hanks et al., 1998]. Mouse Emx1 can rescue brain defects in Drosophila empty spiracles (ems) mutants [Hirth et al., 1995; Hartmann et al., 2000], but Acropora (Anthozoa, Cnidaria) Emx is not able to replace ems in fly brain development [Hartmann et al., 2010].

Based on these results, and in line with Wagner's prediction, it can be concluded that: (1) The character of a 'fore-, mid-, and hindbrain' develops under the control of ChINs comprising otd/Otx, ems/Emx, and en/EN1 genes that are shared in a clade including flies and mice. (2) The failure of Drosophila engrailed to rescue limb formation in Mus EN1 mutants indicates that the appendages of 
mice and flies are not homologous, as has been previously suggested [Panganiban et al., 1997]. (3) Most likely, the epiblast did not exist in the last common ancestor of Drosophila and Mus and is an evolutionary novelty of the lineage leading to Mus [Boyl et al., 2001b]. (4) Drosophila and Acropora ems/Emx genes diverged before the origin of the character 'tripartite brain'. The last conclusion has major implications for the evolutionary origin of the tripartite brain and depends on the position of anthozoans/ cnidarians relative to bilaterally symmetric animals in a phylogenetic tree.

\section{Monophyly Relies on Sister and Out Groups}

A phylogenetic tree is based on evolutionary relationships and can be reconstructed using cladistics. The cladistic concept is relative; it scores characters for their presence and absence and, if present, for their state in each of the taxa of interest [Kitching et al., 1998]. Current cladistics scores morphological as well as molecular characters (i.e. genes, genomes, and developmental pathways) against each other, and the resulting phylogenetic tree is based on sister and out groups in order to be rooted. For example sister groups like arthropods and onychophorans share a common panarthropod ancestor, and together they are a sister group to cycloneuralians (including nematodes), which together belong to the ecdysozoans, which are a sister group to lophotrochozoans (fig. 1). Out groups provide necessary additional information about the origin of a character in sister groups; they are used for character polarity which enables the application of the parsimony criterion in order to infer whether the character is primitive/ancestral or derived [Telford and Budd, 2003].

Monophyly of bilaterally symmetric animals and subsequent interpretations about the origin and evolution of the brain and CNS hinge on the identification of genuine sister and out groups and, thus, on how deep a phylogenetic tree is rooted. Current cladistics is work in progress which is exemplified by the allocation of Cnidaria (but also Acoela) as either a sister or out group to bilaterians [De Robertis, 2008; Martindale and Hejnol 2008]. The ambiguity is caused by mounting evidence suggesting that cnidarians possess genetic toolkits similar to those active in bilaterian axis and cell type specification [Ball et al., 2004], including neurogenesis [Gaillot et al., 2009]. Depending on additional characters and genuine out groups to Cnidaria, this can be interpreted to mean that: (i) cnidarians are de facto bilaterians and a genuine sister group to the rest of bilaterally symmetric animals, namely $\mathrm{P}+\mathrm{D}$, or (ii) cnidarians are a genuine out group to $\mathrm{P}+\mathrm{D}$. It follows that the positioning of Cnidaria has an impact on the positioning of Urbilateria and the origin of a brain and CNS. Cnidarians possess nerve nets and nerve rings but, so far, no evidence of a centralized nervous system has been found [Gaillot et al., 2009]. Several extant P+D also possess a net-like nervous system [Moroz, 2009]. In the first scenario, Urbilateria would be the last common ancestor of both Cnidaria and $\mathrm{P}+\mathrm{D}$, suggesting that a nerve net is a primitive/ancestral character. In the second scenario, Urbilateria would be the last common ancestor of $\mathrm{P}+\mathrm{D}$, and unrelated to Cnidaria, suggesting that the cnidarian nerve net is unrelated to the nerve net of extant bilaterally symmetric animals (the position of Acoela would require further considerations) [De Robertis, 2008; Hejnol and Martindale, 2008; Erwin, 2009].

As mentioned, the presence of genetic toolkits does not necessarily reflect a common origin of characters. The existence of equivalent functions of character identity genes, however, allows inferences as to whether genes diverged before the origin of a character or not [Wagner, 2007]. Mus, but not the cnidarian Acropora Emx gene, can rescue brain defects in Drosophila ems mutants [Hirth et al., 1995; Hartmann et al., 2000; Hartmann et al., 2010], suggesting that Drosophila and Acropora ems/ $E m x$ genes diverged before the origin of the character 'tripartite brain', which is a primitive/ancestral character to Mus and Drosophila. These data suggest that Acropora and the last common ancestor of Mus and Drosophila did not share the character 'tripartite brain'; it may also indicate that the last common ancestor of Cnidaria and $\mathrm{P}+\mathrm{D}$ possessed a nerve net-like nervous system. The latter notion is supported by the functional equivalence of another character identity gene. The cnidarian Hydra achaete scute homolog has proneural activity in Drosophila, can heterodimerize with daughterless, and is able to form ectopic sensory organs in the peripheral nervous system of Drosophila; in addition, it is also able to partially rescue adult external sensory organ formation in viable achaete scute complex mutations [Grens et al., 1995]. Unfortunately, Grens et al. [1995] did not test whether Hydra achaete scute is able to rescue defects in Drosophila achaete scute mutant brain and CNS development.

Together, these data suggest that the morphological character 'tripartite brain' evolved after the Cnidaria$\mathrm{P}+\mathrm{D}$ split. It depends on the placement of cnidarians inor outside the clade Bilateria, whether it is reasonable to propose that Urbilateria already possessed a tripartite brain and probably also a complex CNS, or whether Ur- 


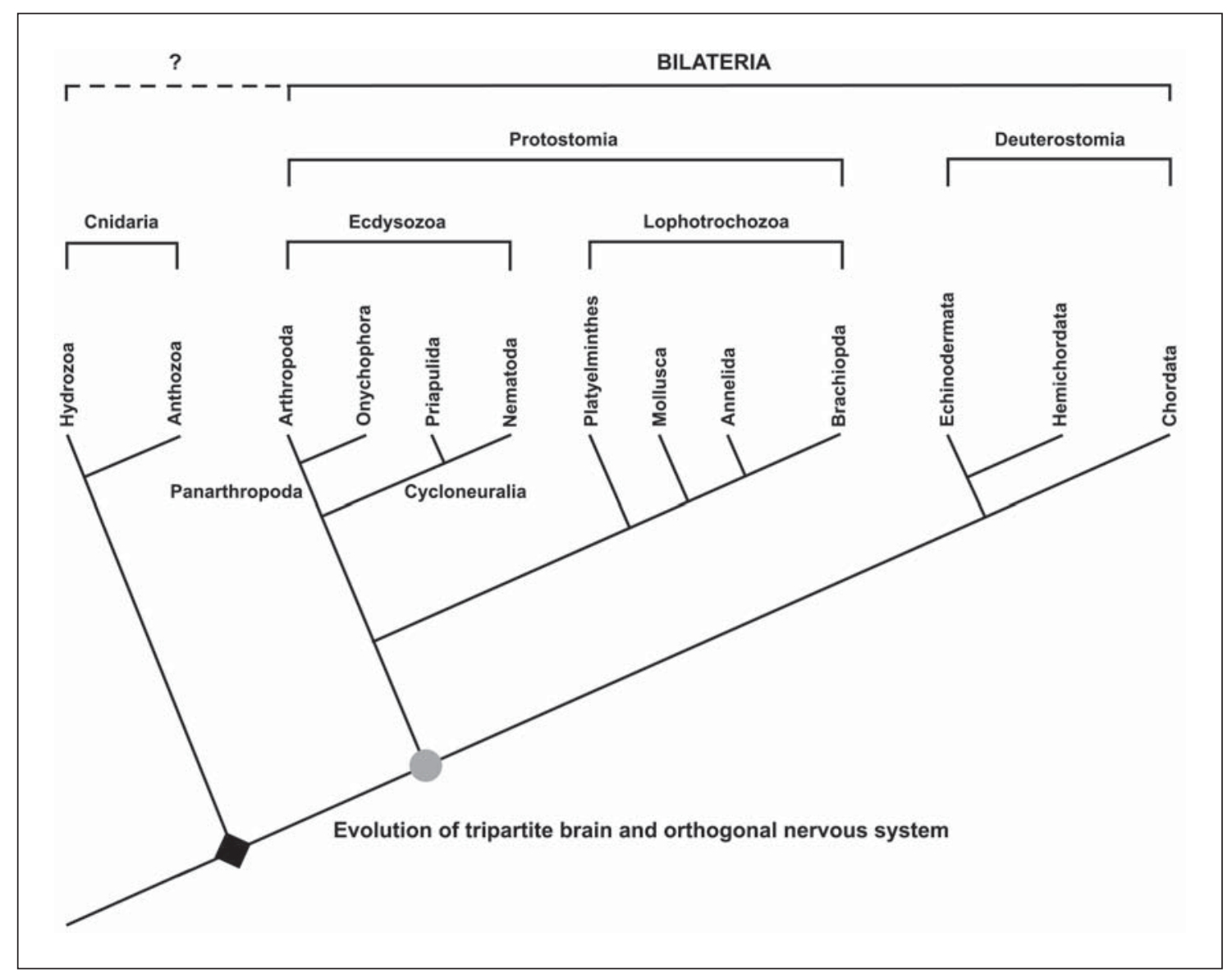

Fig. 1. Evolutionary origin of the tripartite brain. Phylogenetic tree of Bilateria with representative metazoan taxa based on data from Dunn et al. [2008] and Baguñà et al. [2008]. The common classification of Bilateria consists of Protostomia and Deuterostomia $(\mathrm{P}+\mathrm{D})$, but has so far excluded Cnidaria. However, mounting evidence suggests that cnidarians possess genetic toolkits similar to those active in bilaterian axis and cell type specification, including neurogenesis. Based on these features, Cnidaria have been considered bilaterians as well (as indicated by the extended dashed bracket and question mark at the top left). Nodes indicate the last common ancestor. The black square indicates the last common ancestor of Cnidaria and $\mathrm{P}+\mathrm{D}$; the grey circle indicates the last common ancestor of $\mathrm{P}+\mathrm{D}$. Urbilateria and its position in the cladogram depend on the placement of cnidarians as either a sister or out group to $\mathrm{P}+\mathrm{D}$. Independent of the position of Urbilateria, the monophyletic origin and evolution of the tripartite brain and an elongated orthogonal nervous system most likely occurred after the cnidarian-P+D split (black square) and before the ProtoDeuterostomia (P/D) split (grey circle). This scenario is supported by structural homology, character identity networks and functional equivalence of character identity genes; however, it implies the secondary, independent loss of the brain and CNS multiple times during the course of protostomian and deuterostomian evolution. See text for details. bilateria was likely to be 'brainless'. If Cnidaria and $\mathrm{P}+\mathrm{D}$ are true sister groups of a clade Bilateria, it follows that Urbilateria was brainless. Independent of the position of Urbilateria, the above-mentioned data corroborate monophyly of the tripartite brain, as well as its evolutionary origin after the cnidarian- $\mathrm{P}+\mathrm{D}$ split but before the Protostomia-Deuterostomia (P/D) split (fig. 1). This concept, though, is challenged by the fact that several protoand deuterostomians do not possess a brain and complex
CNS. The conundrum is most obvious when the complex CNS of arthropods is compared to that of cycloneuralians, which are supposed to be sister groups. Comparative data for cycloneuralians are scant, except for Caenorhabditis elegans. The nematode does not possess a complex brain and CNS, yet monophyly of the brain is corroborated by functional equivalence of a character identity gene: the ems homolog ceh-2 of C. elegans is able to rescue ems mutant brain defects in Drosophila [Aspöck 
et al., 2003]. These data suggest that C. elegans secondarily lost the character 'tripartite brain' and most likely also a complex CNS, as should be postulated for other brainless proto- and deuterostomians that are monophyletic to Drosophila and Mus.

\section{Secondary Loss of the Brain and CNS in the Course of Evolution}

Monophyly of the tripartite brain and its evolutionary origin after the cnidarian- $\mathrm{P}+\mathrm{D}$ split and before the $\mathrm{P} / \mathrm{D}$ split (fig. 1) implies that the brain and CNS were secondarily reduced and eventually lost multiple times and independently during the course of protostomian and deuterostomian evolution. For the quality and consistency of this conjecture, it is necessary to consider (i) metazoan divergence times, (ii) the likelihood of secondary loss, as illustrated by reconstructed cases, and (iii) the proposal of an experimental paradigm that can test ancestral character identity genes of brainless bilaterians for their potential to control brain development in Drosophila or Mus.

\section{Metazoan Divergence Times}

Molecular clock dates suggest that the cnidarian-P+D split occurred somewhere around 630 million years ago (Mya), the P/D split around $555 \mathrm{Mya}$, and the Arthropoda-Priapulida split around 540 Mya [Peterson et al., 2004; Erwin, 2009]. These estimates suggest that a primitive/ ancestral tripartite brain likely evolved within 75 million years between 630 and 555 Mya and then continued to evolve into taxon- and species-specific characters such as the extant Drosophila and Mus brain. Monophyly implies that ancestral priapulids shared with arthropods the character of a tripartite brain and possibly a segmented CNS. Fossil evidence supports ancestral segmentation in priapulids (e.g. Markuelia) [Dong et al., 2005] even though extant priapulids are nonsegmented and their CNS is 'only' composed of a nerve ring and a single ventral cord running the length of the body. Scaled against molecular clock dates, monophyly of the tripartite brain suggests that the derived character of the priapulid brain and CNS would have had several hundred million years' time for its secondary modification. Such a scale of divergence time can be extrapolated to other taxa as well, and implies that also other extant brainless $\mathrm{P}+\mathrm{D}$ would have had several hundred million years' time for the secondary, independent modification and eventual loss of a tripartite brain and CNS.
The Likelihood of Secondary Loss

Comparative developmental genetics and phylogenomics reveal that morphological evolution is most likely driven by gene duplication and gene loss, together with changes in differential gene regulation, including mutations in cis-regulatory elements of pleiotropic developmental regulatory genes [Carroll, 2008]. These genetic modifications can account not only for the acquisition of novel morphological characters but also for the modification and eventual loss of a morphological character. The latter is illustrated by limbless tetrapods, such as whales, snakes, and flightless birds. Limbless tetrapods are descended from limbed ancestors, and limblessness has been shown to be polygenic, involving pleiotropic regulatory genes that act as modifiers to suppress limb development [Lande, 1978]. In snakes, for example, differential regulation of HoxC genes accounts for the failure to activate the signaling pathways required for proper limb development, eventually leading to limbless snakes [Cohn and Tickle, 1999]. Independent reduction and limb loss in tetrapods occurred repeatedly over several millions of years for lizards [Kearney and Stuart, 2004], and over 1012 or up to 20 million years for whales [Bejder and Hall, 2002].

The secondary loss of morphological characters is also exemplified in fish. In different natural populations of threespined stickleback fish, the secondary loss of the pelvis occurred through regulatory mutations deleting a tissue-specific enhancer of the Pituitary homeobox transcription factor 1 (Pitx1) gene [Chan et al., 2010]. The selective pressures causing secondary loss can be manifold, including energy limitation and environmental constraints which are most obvious for nervous system structures that are characterized by high energy consumption. For example, populations of cave fish have undergone convergent eye loss at least 3 times within the last 1 million years, whereas populations that continuously lived on the surface retained their eyes [Niven and Laughlin, 2008]. These examples illustrate that the secondary loss of a morphological character can occur repeatedly during the course of evolution within a time frame of million years. In comparison, monophyly of the tripartite brain calibrated by metazoan divergence times suggests that extant brainless $\mathrm{P}+\mathrm{D}$ would have had several hundred million years' time, possibly from 555 Mya onwards, for the secondary modification and eventual loss of an ancestral/primitive tripartite brain and CNS, the mechanisms of which remain unknown. 


\section{Can Genes from Brainless Bilateria Rescue Brain Development?}

The secondary, independent loss of the brain and CNS multiple times during the course of protostomian and deuterostomian evolution is a conjecture that can be tested experimentally. Wagner's prediction states that the phenotype caused by knockdown of a character identity gene can be rescued with a gene from the clade that shares a particular character, but not by genes from species that diverged before the origin of this character [Wagner, 2007]. Monophyly of the tripartite brain implies that extant brainless $\mathrm{P}+\mathrm{D}$ species were once able to develop a primitive/ancestral tripartite brain; therefore, these brainless species should possess ChINs for the development and specification of a tripartite brain, unless they have secondarily lost the necessary genes during the course of evolution. The potential functional equivalence of character identity genes in brain development can be tested in those cases where brainless $\mathrm{P}+\mathrm{D}$ species have retained the ChINs or at least a character identity gene. Thus, genes from species that have secondarily lost the character tripartite brain but have retained, for example,
otd/Otx genes with an archetypical 'brain function' might be able to rescue, at least in part, brain phenotypes in Drosophila otd or Mus Otx2 mutants. These experiments are feasible and can be tested as homologs of character identity genes controlling tripartite brain development have been identified in brainless $\mathrm{P}+\mathrm{D}$ species. It will be interesting to see whether Otx or Engrailed genes from brainless brachiopods [Holland et al., 1991] or echinoderms like sea cucumber [Shoguchi et al., 2000] are able to substitute their Drosophila or Mus homologs in the development and specification of a tripartite brain.

\section{Acknowledgements}

The author thanks Dickon Humphrey and Beate Haege for their comments on the manuscript. Work in the author's laboratory is supported by grants from the UK Medical Research Council (G070149), the Royal Society (Hirth/2007/02), Parkinson's UK (G-0714), the Motor Neurone Disease Association (Hirth/ Oct07/6233), and the King's College NHS Medical Research Trust (JRC19/2007).

\section{References}

-Acampora D, Boyl PP, Signore M, Martinez-Barbera JP, Ilengo C, Puelles E, Annino A, Reichert H, Corte G, Simeone A (2001): OTD/OTX2 functional equivalence depends on $5^{\prime}$ and $3^{\prime}$ UTR-mediated control of Otx2 mRNA for nucleo-cytoplasmic export and epiblast-restricted translation. Development 128:4801-4813.

-Arendt D, Denes AS, Jékely G, Tessmar-Raible K (2008): The evolution of nervous system centralization. Philos Trans R Soc Lond B Biol Sci 363:1523-1528.

-Aspöck G, Ruvkun G, Bürglin TR (2003): The Caenorhabditis elegans ems class homeobox gene ceh-2 is required for M3 pharynx motoneuron function. Development 130:33693378.

Baguñà J, Martinez P, Paps J, Riutort M (2008): Back in time: a new systematic proposal for the Bilateria. Philos Trans R Soc Lond B Biol Sci 363:1481-1491.

Ball EE, Hayward DC, Saint R, Miller DJ (2004): A simple plan: cnidarians and the origins of developmental mechanisms. Nat Rev Genet 5:567-577.

Bejder L, Hall BK (2002): Limbs in whales and limblessness in other vertebrates: mechanisms of evolutionary and developmental transformation and loss. Evol Dev 4:445458 .
Boyl PP, Signore M, Annino A, Barbera JP, Acampora D, Simeone A (2001a): Otx genes in the development and evolution of the vertebrate brain. Int J Dev Neurosci 19:353-363.

Boyl PP, Signore M, Acampora D, Martinez-Barbera JP, Ilengo C, Annino A, Corte G, Simeone A (2001b): Forebrain and midbrain development requires epiblast-restricted Otx2 translational control mediated by its $3^{\prime}$ UTR Development 128:2989-3000.

Carroll SB (2008): Evo-devo and the expanding evolutionary synthesis: a genetic theory of morphological evolution. Cell 134:25-36.

Chan YF, Marks ME, Jones FC, Villarreal G Jr, Shapiro MD, Brady SD, Southwick AM, Absher DM, Grimwood J, Schmutz J, Myers RM, Petrov D, Jónsson B, Schluter D, Bell MA, Kingsley DM (2010): Adaptive evolution of pelvic reduction in sticklebacks by recurrent deletion of a Pitx1 enhancer. Science 327:302-305.

Christiaen L, Jaszczyszyn Y, Kerfant M, Kano S, Thermes V, Joly JS (2007): Evolutionary modification of mouth position in deuterostomes. Semin Cell Dev Biol 18:502-511.

Cohn MJ, Tickle C (1999): Developmental basis of limblessness and axial patterning in snakes. Nature 399:474-479.

Darwin C (1859): On the origin of species by means of natural selection, or the preservation of favoured races in the struggle for life. London, J Murray.
Denes AS, Jékely G, Steinmetz PR, Raible F, Snyman H, Prud'homme B, Ferrier DE, Balavoine G, Arendt D (2007): Molecular architecture of annelid nerve cord supports common origin of nervous system centralization in bilateria. Cell 129:277-288.

De Robertis EM (2008): Evo-devo: variations on ancestral themes. Cell 132:185-195.

Dong XP, Donoghue PC, Cunningham JA, Liu JB, Cheng H (2005): The anatomy, affinity, and phylogenetic significance of Markuelia. Evol Dev 7:468-482.

$>$ Dunn CW, Hejnol A, Matus DQ, Pang K, Browne WE, Smith SA, Seaver E, Rouse GW, Obst M, Edgecombe GD, Sørensen MV, Haddock SH, Schmidt-Rhaesa A, Okusu A, Kristensen RM, Wheeler WC, Martindale MQ, Giribet $\mathrm{G}$ (2008): Broad phylogenomic sampling improves resolution of the animal tree of life. Nature 452:745-749.

Erwin DH (2009): Early origin of the bilaterian developmental toolkit. Philos Trans R Soc Lond B Biol Sci 364:2253-2261.

Galliot B, Quiquand M, Ghila L, de Rosa R, Miljkovic-Licina M, Chera S (2009): Origins of neurogenesis, a cnidarian view. Dev Biol 332:2-24.

Goridis C, Rohrer H (2002): Specification of catecholaminergic and serotonergic neurons. Nat Rev Neurosci 3:531-541. 
-Grens A, Mason E, Marsh JL, Bode HR (1995): Evolutionary conservation of a cell fate specification gene: the Hydra achaete-scute homolog has proneural activity in Drosophila. Development 121:4027-4035.

-Hanesch U, Fischbach KF, Heisenberg M (1989): Neuronal architecture of the central complex in Drosophila melanogaster. Cell Tissue Res 257:343-366.

- Hanks MC, Loomis CA, Harris E, Tong CX, Anson-Cartwright L, Auerbach A, Joyner A (1998): Drosophila engrailed can substitute for mouse Engrailed 1 function in mid-hindbrain, but not limb development. Development 125:4521-4530.

-Hartmann B, Hirth F, Walldorf U, Reichert H (2000): Expression, regulation and function of the homeobox gene empty spiracles in brain and ventral nerve cord development of Drosophila. Mech Dev 90:143-153.

- Hartmann B, Müller M, Hislop NR, Roth B, Tomljenovic L, Miller DJ, Reichert H (2010): Coral emx-Am can substitute for Drosophila empty spiracles function in head, but not brain development. Dev Biol 340:125-133.

-Hejnol A, Martindale MQ (2008): Acoel development supports a simple planula-like urbilaterian. Philos Trans R Soc Lond B Biol Sci 363:1493-1501.

- Hirth F, Kammermeier L, Frei E, Walldorf U, Noll M, Reichert H (2003): An urbilaterian origin of the tripartite brain: developmental genetic insights from Drosophila. Development 130:2365-2373.

- Hirth F, Reichert H (1999): Conserved genetic programs in insect and mammalian brain development. Bioessays 21:677-684.

Hirth F, Reichert H (2007): Basic nervous system types: one or many? in Kaas JH (ed): Evolution of Nervous Systems. Amsterdam, Academic Press, vol 1, pp 55-72.

- Hirth F, Therianos S, Loop T, Gehring WJ, Reichert H, Furukubo-Tokunaga K (1995): Developmental defects in brain segmentation caused by mutations of the homeobox genes orthodenticle and empty spiracles in Drosophila. Neuron 15:769-778.

Holland ND (2003): Early central nervous system evolution: an era of skin brains? Nat Rev Neurosci 4:1-11.

-Holland PW, Williams NA, Lanfear J (1991): Cloning of segment polarity gene homologues from the unsegmented brachiopod Terebratulina retusa (Linnaeus). FEBS Lett 291:211-213.
Kearney M, Stuart BL (2004): Repeated evolution of limblessness and digging heads in worm lizards revealed by DNA from old bones. Proc Biol Sci 271:1677-1683.

Kitching IJ, Humphries C, Williams D, Forey PL (1998): Cladistics: The Theory and Practice of Parsimony Analysis. Oxford, Oxford University Press.

Kong EC, Woo K, Li H, Lebestky T, Mayer N, Sniffen MR, Heberlein U, Bainton RJ, Hirsh J, Wolf FW (2010): A pair of dopamine neurons target the D1-like dopamine receptor DopR in the central complex to promote ethanol-stimulated locomotion in Drosophila. PLoS One 5:e9954.

Kozmik Z (2005): Pax genes in eye development and evolution. Curr Opin Genet Dev 15 430-438.

Lande R (1978): Evolutionary mechanisms of limb loss in tetrapods. Evolution 32:73-92.

Leuzinger S, Hirth F, Gerlich D, Acampora D, Simeone A, Gehring WJ, Finkelstein R, Furukubo-Tokunaga K, Reichert H (1998): Equivalence of the fly orthodenticle gene and the human OTX genes in embryonic brain development of Drosophila. Development 125:1703-1710.

Lorenz KZ (1974): Analogy as a source of knowledge. Science 185:229-234.

Lowe CJ, Terasaki M, Wu M, Freeman RM Jr, Runft L, Kwan K, Haigo S, Aronowicz J, Lander E, Gruber C, Smith M, Kirschner M, Gerhart J (2006): Dorsoventral patterning in hemichordates: insights into early chordate evolution. PLoS Biol 4:e291.

Martindale MQ, Hejnol A (2009): A developmental perspective: changes in the position of the blastopore during bilaterian evolution. Dev Cell 17:162-174.

Meinhardt H (2002): The radial-symmetric hydra and the evolution of the bilateral body plan: an old body became a young brain. Bioessays 24:185-191.

Melcher C, Pankratz MJ (2005): Candidate gus tatory interneurons modulating feeding behavior in the Drosophila brain. PLoS Biol 3:e305

Mindell DP, Meyer A (2001): Homology evolving. Trends Ecol Evol 16:434-440.

Moroz LL (2009): On the independent origins of complex brains and neurons. Brain Behav Evol 74:177-190.

Neuser K, Triphan T, Mronz M, Poeck B, Strauss R (2008): Analysis of a spatial orientation memory in Drosophila. Nature 453:12441247.
Nichols CD (2007): 5-HT2 receptors in Drosophila are expressed in the brain and modulate aspects of circadian behaviors. Dev Neurobiol 67:752-763.

Niven JE, Laughlin SB (2008): Energy limitation as a selective pressure on the evolution of sensory systems. J Exp Biol 211:1792-804.

-Nomaksteinsky M, Röttinger E, Dufour HD, Chettouh Z, Lowe CJ, Martindale MQ, Brunet JF (2009): Centralization of the deuterostome nervous system predates chordates. Curr Biol 19:1264-1269.

Northcutt RG (2010): Cladistic analysis reveals brainless urbilateria. Brain Behav Evol 76:1-2.

Panganiban G, Irvine SM, Lowe C, Roehl H, Corley LS, Sherbon B, Grenier JK, Fallon JF, Kimble J, Walker M, Wray GA, Swalla BJ, Martindale MQ, Carroll SB (1997): The origin and evolution of animal appendages. Proc Natl Acad Sci USA 94:5162-5166.

Peterson KJ, Lyons JB, Nowak KS, Takacs CM, Wargo MJ, McPeek MA (2004): Estimating metazoan divergence times with a molecular clock. Proc Natl Acad Sci USA 101:65366541.

Prakash N, Wurst W (2004): Specification of midbrain territory. Cell Tissue Res 318:5-14.

-Shoguchi E, Harada Y, Numakunai T, Satoh N (2000): Expression of the otx gene in the ciliary bands during sea cucumber embryogenesis. Genesis 27:58-63.

$\checkmark$ Shubin N, Tabin C, Carroll S (2009): Deep homology and the origins of evolutionary novelty. Nature 457:818-823.

Telford MJ, Budd GE (2003): The place of phylogeny and cladistics in Evo-Devo research. Int J Dev Biol 47:479-490.

Thor S, Thomas JB (2002): Motor neuron specification in worms, flies and mice: conserved and 'lost' mechanisms. Curr Opin Genet Dev 12:558-564.

Travers JB, Travers SP, Norgren R (1987): Gustatory neural processing in the hindbrain. Annu Rev Neurosci 10:595-632.

-Wada H, Saiga H, Satoh N, Holland PW (1998): Tripartite organization of the ancestral chordate brain and the antiquity of placodes: insights from ascidian Pax-2/5/8, Hox and Otx genes. Development 125:1113-1122.

Wagner GP (2007): The developmental genetics of homology. Nat Rev Genet 8:473-479.

Wurst W, Bally-Cuif L (2001): Neural plate patterning: upstream and downstream of the isthmic organizer. Nat Rev Neurosci 2:99108 